\title{
Electroconvulsive Therapy: a Video-Based Educational Resource Using Standardized Patients
}

\author{
Brandon Kitay ${ }^{1} \cdot$ Andrés Martin $^{2}$ (D) - Julie Chilton ${ }^{2} \cdot$ Doron Amsalem $^{3} \cdot$ Robbert Duvivier $^{4} \cdot$ Matthew Goldenberg $^{1}$
}

Received: 20 April 2020 / Accepted: 19 July 2020 / Published online: 4 August 2020

(C) Academic Psychiatry 2020

\begin{abstract}
Objective Video-based depictions of electroconvulsive therapy (ECT) can be useful for educational purposes, but many of the readily available resources may worsen already stigmatized views of the procedure. Educators' common reliance on such material highlights the paucity of equipoised depictions of modern ECT well suited for the training of health professionals. The authors developed and tested a new educational module enhanced by videotaped depictions of a simulated patient undergoing the consent, treatment, recovery, and follow-up phases of ECT.

Methods The didactic intervention interspersed 7 short video clips (totaling $14 \mathrm{~min}$ ) into a 55 -min lecture on treatment-resistant depression. The session, part of an intensive course of preclinical psychiatry, was delivered online through synchronous videoconferencing with Zoom. The primary outcome measure was change in the Questionnaire on Attitudes and Knowledge of ECT (QuAKE).

Results Fifty-three out of $63(87 \%)$ eligible second-year medical students completed assessments at baseline and after exposure to the didactic intervention. QuAKE scores improved between baseline and endpoint: the Attitudes composite increased from $49.4 \pm 6.1$ to $59.1 \pm 5.7$ (paired $t 10.65, p<0.001$, Cohen's $d 0.69$ ), and the Knowledge composite from $13.3 \pm 1.2$ to $13.9 \pm 0.8$ (paired $t 3.97, p<0.001$, Cohen's $d 0.23$ ).

Conclusions These video-based educational materials proved easy to implement in the virtual classroom, were amenable to adaptation by end-use instructors, were well received by learners, and led to measurable changes in students' knowledge of and attitudes toward ECT.
\end{abstract}

Keywords Electroconvulsive therapy (ECT) - Curriculum development $\cdot$ Teaching materials $\cdot$ Standardized patient (SP) . Synchronous videoconferencing $\cdot$ Stigma

Electroconvulsive therapy (ECT) remains one of the safest and most effective treatments in psychiatric practice. However, ECT utilization remains low relative to the need for effective interventions in "treatment-refractory" psychiatric patients. Low rates of utilization have been attributed to a complex set of structural, cultural, and region-specific legislative barriers $[1,2]$. At the level of the practitioner, lack of knowledge about the efficacy of the procedure and/or negative

Andrés Martin andres.martin@yale.edu

1 Yale School of Medicine, New Haven, CT, USA

2 Child Study Center, Yale School of Medicine, New Haven, CT, USA

3 Tel Aviv University Faculty of Medicine, Ramat Aviv, Israel

4 University Medical Center Groningen, Groningen, The Netherlands attitudes regarding ECT remain significant barriers toward making referrals or recommending ECT as an appropriate treatment. Professional organizations such as the American Psychiatric Association (APA) [3] and the Association of Directors of Medical Student Education in Psychiatry (ADMSEP) [4] have highlighted knowledge about ECT in their recommended learning objectives for medical students, but ECT education in health professional programs is not standardized and remains heterogeneous $[5,6]$. In settings where there is limited local expertise in ECT or "interventional psychiatry" [7], trainees may have few if any opportunities to witness the procedure in person.

Many healthcare trainees in the USA and internationally harbor negative attitudes toward ECT $[8,9]$. Popular media, including movies, television, and the Internet, are common sources of information - and misinformation-about ECT. A systematic review of media portrayals of ECT showed that 
a majority of those depictions are exaggerated, frightening, and only loosely related to the actual practice [10]. These cultural influences are as hard to ignore as they are inaccurate. ECT is a highly effective intervention, and patients who have undergone the treatment report positive impacts on their related knowledge and attitudes toward the procedure [11].

A growing evidence base suggests that even brief educational interventions, including videotaped observation of modern treatment $[12,13]$, can significantly improve perceptions of ECT among students [14-16] and prospective patients [17]. Videotaped depictions of ECT have logistic advantages as well, including ease of access, consistency of the depiction used for teaching, and potential suitability for a flipped curriculum approach.

As helpful as video-based depictions of ECT can be for educational purposes, many of the more readily available sources in television [10], film [9, 10], or YouTube [18] can serve to further entrench already stigmatized views of the procedure without appropriate context and opportunity for discussion. Educators' common reliance on such material highlights the paucity of widely available and equipoised depictions of modern ECT well suited for the training of health professionals.

Our goal was to develop and test a new educational module enhanced by videotaped depictions of a simulated patient undergoing the consent, treatment, recovery, and follow-up phases of ECT. Care was taken to provide factual, nonstigmatizing depiction of the process. Our objectives were to improve knowledge and attitudes about ECT in various training settings, with the ultimate hope that participating trainees will, in the future, be aware of the availability of ECT and make appropriate referrals to and proper utilization of this highly effective but often overlooked treatment modality.

\section{Methods}

\section{Participants and Synchronized Videoconferencing Delivery}

Participants were second-year medical students at the TelAviv University Faculty of Medicine's New York State Program. Starting in 2016, the Program's pre-clinical psychiatry course has been led by one of the authors (AM), with two others joining as faculty since 2018 (JC) and 2019 (DA). The 30-h course, spread over 5 days, includes lectures, workshops, team-based learning, flipped classroom activities, and videoenhanced clinical discussions. The fifth iteration of this annual course was scheduled to be delivered in person from March 31 through April 7, 2020.

The COVID-19 pandemic resulted in the declaration of a national state of emergency in Israel on March 19. The course was initially canceled, but later rescheduled to an online, video-conferencing platform (Zoom, San Jose, CA). This approach permitted the course to be offered virtually through synchronous content delivery and effective interaction between faculty and students.

\section{Educational Intervention}

We used the video-based components of a new ECT educational module developed by two of the authors (BK and $\mathrm{MG}$ ) as part of a broader project funded by the Association for Directors of Medical Student Education in Psychiatry (ADMSEP). The overall project includes didactic slides interwoven with videos of the informed consent process, the procedure itself, and recovery and follow-up phases. All videobased depictions provide a detailed, factual, and present-day representation of ECT. The videos incorporate providers routinely involved in the provision of ECT as part of an interventional psychiatry team at Yale New Haven Hospital (i.e., an interventional psychiatrist, an anesthesiologist, and a nurse). The patient ("Annie") and family member (daughter) in the video are professional actors hired through the Standardized Patient Program of the Yale School of Medicine's Teaching and Learning Center, for which one of the authors (AM) serves as director. The actors are experienced in medical settings and followed accepted best practices for standardized patients [19].

The video-based component of the project consists of four modules, with a total duration of $36 \mathrm{~min}$. The complete footage is currently being incorporated into an online educational module about ECT that will be published in the ADMSEP website (admsep.org). For the purposes of this course, we edited the material down to seven short clips, with a total duration of $14 \mathrm{~min}$. The different video components and their respective lengths are described in Table 1. We interspersed these short clips into a 55-min lecture on treatmentresistant depression.

\section{Outcome Measure}

Participants provided demographic information and repeated a standardized instrument at baseline and endpoint. The Questionnaire on Attitudes and Knowledge of ECT (QuAKE) [20] is a widely used instrument that has been shown to have good internal consistency (Cronbach $\alpha, 0.91$ ) and split-half reliability in a sample of mental health professionals (Guttman split-half score, 0.92). The QuAKE has two components: (1) Attitudes, for which respondents indicate how strongly they agree or disagree on a 5-point Likert scale with 16 positive and negative statements about ECT (for a maximum score of $5 \times 16=80$ ) - some of the items are reverse-coded to prevent response acquiescence bias; and (2) Knowledge, a list of 16 factual items with a forced true (1) or false (0) response. 
Table 1 ECT video modules and edited clips

\begin{tabular}{|c|c|c|c|c|}
\hline \multicolumn{2}{|l|}{ Version (total length) } & \multicolumn{3}{|l|}{ Time } \\
\hline Modules (36:24) & Edited clips (14:22) & Start & Finish & Duration \\
\hline \multirow[t]{2}{*}{ 1. Patient interview and symptoms } & & 00:00 & $10: 51$ & $10: 51$ \\
\hline & 1a. Clinical presentation & 00:00 & $02: 45$ & 02:45 \\
\hline \multirow[t]{3}{*}{ 2. Informed consent and treatment options } & & 00:00 & $11: 44$ & $11: 44$ \\
\hline & 2a. Treatment, offered & 02:19 & $03: 47$ & $01: 28$ \\
\hline & 2b. Treatment, explained & 09:40 & $12: 15$ & $02: 35$ \\
\hline \multirow[t]{4}{*}{ 3. The procedure } & & 00:00 & $05: 33$ & $05: 33$ \\
\hline & 3a. Anesthesiologist & 00:10 & 01:27 & $01: 17$ \\
\hline & 3b. Going under & $01: 28$ & 03:21 & $01: 53$ \\
\hline & 3c. The procedure & $01: 54$ & 04:04 & $02: 10$ \\
\hline \multirow[t]{2}{*}{ 4. Post-treatment interview } & & 00:00 & 08:16 & $08: 16$ \\
\hline & 4a. After nine treatments & 00:00 & $02: 14$ & $02: 14$ \\
\hline
\end{tabular}

All time measures are denoted as minutes:seconds; each of the four modules is a separate video file starting at time stamp 00:00

\section{Ethics Approval}

Before starting data collection, we obtained institutional review board approval from the Yale Human Investigations Committee (Protocol no. 2000026843) and the Tel-Aviv University Faculty of Medicine (Protocol no. 0000946-1). The study was deemed exempt of review, with completion of the survey representing tacit consent. Students were encouraged to participate but informed that their participation was neither mandatory nor relevant to their performance evaluation for the course. They were notified that results of the surveys would not be accessible to faculty responsible for their course evaluations. In order to track individuals' responses over time, each student provided a deidentified and anonymous study code.

\section{Data Collection and Statistical Analysis}

Students completed surveys through their preferred, WiFienabled personal devices during dedicated classroom time on two dates in early April 2020. We collected information securely through Qualtrics (Provo, UT), and analyzed data using SPSS version 25 (Armonk, NY).

We compared differences from baseline to endpoint using paired $t$ tests and Cohen's $d$ effect sizes for continuous variables (Attitudes and Knowledge global scores). We next compared the 16 individual Attitudes items, adjusting for multiple comparisons through a Bonferroni correction, which yielded a corrected $p$ value significance threshold of $0.05 / 16=0.003$. We then used chi-square tests for the 16 categorical Knowledge items, relying on McNemar's test to examine changes before and after the intervention. McNemar's test is used to determine differences on a dichotomous dependent variable between two related groups and is used to analyze pre-post study designs [21].

\section{Results}

All students in the second-year class $(n=63)$ were invited to participate. Table 2 summarizes characteristics of the $61 \mathrm{stu}-$ dents (97\%) who completed the baseline assessment. Most students were still in Israel during the course, but one-third had gone back to their homes in the USA or Canada; these students took the course synchronously, but with a 7- or 11-h difference (EST 23\%, or PST 7\%, respectively). All content was taught in English. Many had experiences with mental illness: in a friend or relative (77\%), or personally (18\%). Students reported substantial interest in psychiatry as a possible specialty, with only $28 \%$ of them ruling it out altogether.

Fifty-three students (84\% of the class) completed endpoint assessments that could be matched to baseline surveys. (Data for eight students could not be used at endpoint as their deidentified codes could not be matched to baseline responses.) There were no differences in baseline characteristics between included and excluded respondents $(p>0.05$ for all comparisons). QuAKE Attitudes and Knowledge composites improved between baseline and endpoint among the 53 respondents with matched data $(p<0.001)$ (Table 3). Mean Attitudes score increased from 62 to $74 \%$ after exposure to the didactic intervention (for a moderate effect size of 0.69), and mean Knowledge score increased from 83 to $87 \%$ (for a small effect size of 0.23).

In order to better understand the specific components driving these changes, we conducted a secondary item-level analysis. Table 4 summarizes these findings, rank ordered by the 
Table 2 Descriptive characteristics of second-year medical students $(n=61)$

\begin{tabular}{|c|c|c|}
\hline Characteristic & $n$ & $\%$ \\
\hline \multicolumn{3}{|l|}{ Sex } \\
\hline Female & 28 & 46 \\
\hline Male & 33 & 54 \\
\hline \multicolumn{3}{|l|}{ Age } \\
\hline 24 and under & 21 & 34 \\
\hline $25-29$ & 40 & 66 \\
\hline \multicolumn{3}{|c|}{ Geographic location } \\
\hline Israel & 43 & 70 \\
\hline Elsewhere & 18 & 30 \\
\hline \multicolumn{3}{|c|}{ Time zone (UTC) } \\
\hline Israel $(+3)$ & 43 & 70 \\
\hline $\operatorname{EST}(-5)$ & 14 & 23 \\
\hline $\operatorname{PST}(-8)$ & 4 & 7 \\
\hline \multicolumn{3}{|c|}{ Experience with mental illness } \\
\hline \multicolumn{3}{|c|}{ Have a friend or relative diagnosed with } \\
\hline Yes & 47 & 77 \\
\hline No & 14 & 23 \\
\hline \multicolumn{3}{|c|}{ Ever been diagnosed with } \\
\hline Yes & 11 & 18 \\
\hline No & 50 & 82 \\
\hline \multicolumn{3}{|c|}{ Would consider specializing in psychiatry } \\
\hline Yes & 13 & 21 \\
\hline Maybe & 31 & 51 \\
\hline No & 17 & 28 \\
\hline
\end{tabular}

UTC, Coordinated Universal Time

degree of change from baseline to endpoint. Twelve of the sixteen attitudinal items improved, particularly around the indications for the treatment in current psychiatric clinical practice. Ten of the sixteen knowledge items improved after the intervention. This resulted in the rectification of several misconceptions the students had about ECT, toward: (1) brain damage, permanent memory impairment, and fractures are not common side effects; (2) there is a robust evidence base for the use of ECT; and (3) the treatment is not contraindicated during pregnancy or in the setting of dementia.

Finally, sixteen students ( $30 \%$ of those with complete data) provided responses to our optional question about the session in general, and about its video-based component in particular. Comments were uniformly positive, and representative verbatim quotes included the following: "I appreciated learning about the efficacy of ECT for treatment and the session changed my view about the subject in a positive way"; "the video helped me understand it better as a treatment. I had a different picture of it in my mind before the session"; "informative and helpful to be walked through the steps of ECT"; "having patient cases (like Annie's getting ECT) really brings the concepts and ideas to life - and I remember them better"; "very helpful to demystify ECT. It is helpful to see it and not just read about it because you feel like you can actually understand what is happening and what the experience is like for the patient. It doesn't look so scary after all"; and "the session was very informative and was approached with sensitivity."

\section{Discussion}

We derive two main sets of conclusions from our study: one about its didactic content, another from its means of delivery. After separately addressing each of the two, we identify limitations and next steps.

\section{Content: Video-Enhanced Education on ECT}

Our video-based educational materials proved easy to implement in the classroom, were amenable to adaptation by enduse instructors, were well received by learners, and led to measurable changes in their knowledge and attitude scores pertaining to underlying content.

With regard to ease of implementation and adaptability of content, these video-based materials can be edited to address the specific needs of different groups of learners. In this instance, we addressed second-year medical students during a 1-h slot in their pre-clinical psychiatry curriculum. To that
Table 3 Main outcomes of ECT training intervention $(n=53)$

\begin{tabular}{|c|c|c|c|c|c|c|c|}
\hline \multirow[b]{2}{*}{ Scale } & \multicolumn{2}{|l|}{ Pre } & \multicolumn{2}{|l|}{ Post } & \multirow{2}{*}{$\begin{array}{l}\text { Difference } \\
\text { Mean }(95 \% \mathrm{CI})\end{array}$} & \multicolumn{2}{|l|}{ Statistic } \\
\hline & Mean & $\mathrm{SD}$ & Mean & SD & & Paired $t^{\mathrm{a}}$ & Cohen's $d$ \\
\hline \multicolumn{8}{|l|}{ QuAKE } \\
\hline Attitudes & 49.4 & 6.1 & 59.1 & 5.7 & $9.7(7.9,11.5)$ & $10.65 * * *$ & 0.69 \\
\hline Knowledge & 13.3 & 1.2 & 13.9 & 0.8 & $1.3(0.6,4.0)$ & $3.97 * * *$ & 0.23 \\
\hline
\end{tabular}

QuAKE, Questionnaire on Attitudes and Knowledge of ECT

${ }^{\mathrm{a}} \mathrm{df}=52$

$* * * p<0.001$ 
Table 4 Change in QuAKE item scores between baseline and endpoint

\begin{tabular}{|c|c|c|c|c|c|}
\hline Domain & Item & Stem & Pre & Post & Statistic \\
\hline \multirow[t]{17}{*}{ Attitudes ${ }^{\text {a }}$} & & & $\begin{array}{l}\text { Mean } \\
\text { (SD) }\end{array}$ & $\begin{array}{l}\text { Mean } \\
\text { (SD) }\end{array}$ & Paired $t$ \\
\hline & 16 & ${ }^{\dagger}$ In this day of modern medicine ECT should be banned & $1.4(0.6)$ & $2.6(0.8)$ & $9.62 * * *$ \\
\hline & 5 & ECT is usually used appropriately & $1.8(0.7)$ & $2.8(0.7)$ & $9.09 * * *$ \\
\hline & 3 & ECT is more likely to be beneficial than harmful & $1.7(0.7)$ & $2.9(0.7)$ & $8.68 * * *$ \\
\hline & 1 & Would consider ECT as treatment option for friend/relative & $1.7(0.7)$ & $2.9(1.0)$ & $8.25 * * *$ \\
\hline & 4 & ECT is unlikely to cause brain damage & $1.9(0.9)$ & $3.1(0.8)$ & $7.75 * * *$ \\
\hline & 14 & ${ }^{\dagger}$ There is no real proof that ECT works & $1.6(0.6)$ & $2.4(0.7)$ & $7.42 * * *$ \\
\hline & 13 & ${ }^{\dagger} \mathrm{ECT}$ is a cruel treatment & $1.5(0.6)$ & $2.5(0.9)$ & $6.97 * * *$ \\
\hline & 15 & ${ }^{\dagger}$ Although the patient may recover from ECT, he/she will never be the same for having it & $1.9(0.8)$ & $2.8(0.8)$ & $5.45 * * *$ \\
\hline & 7 & Patients are sufficiently informed about likely effects and side-effects & $2.0(1.0)$ & $2.8(0.8)$ & $5.25 * * *$ \\
\hline & 9 & ${ }^{\dagger}$ ECT should only be used as a last resort & $2.8(1.1)$ & $3.5(0.8)$ & $5.11 * * *$ \\
\hline & 8 & $\begin{array}{l}\dagger \text { Imagining myself having ECT is more worrying than the thought of having surgery for } \\
\text { appendicitis }\end{array}$ & $2.8(1.1)$ & $3.6(1.3)$ & $4.44 * * *$ \\
\hline & 11 & ${ }^{\dagger}$ Psychiatrists use ECT because they do not know how else to treat the patient & $2.2(1.0)$ & $2.9(1.0)$ & $4.12 * * *$ \\
\hline & 6 & Psychiatrists take other members of staffs' views into account when deciding on ECT & $2.1(1.0)$ & $2.6(0.7)$ & 2.82 \\
\hline & 12 & ${ }^{\dagger} \mathrm{I}$ find the most disturbing aspect of ECT to be the use of electricity & $2.6(1.0)$ & $2.9(1.0)$ & 1.88 \\
\hline & 2 & ${ }^{\dagger}$ Major surgery is more dangerous than ECT & $3.6(1.4)$ & $3.3(1.0)$ & -1.67 \\
\hline & 10 & ${ }^{\dagger}$ It is the induced seizure that I find most worrying about ECT & $3.1(1.2)$ & $3.3(0.9)$ & 0.93 \\
\hline \multirow[t]{17}{*}{ Knowledge $^{\mathrm{b}}$} & & & $n(\%)$ & $n(\%)$ & $x^{2}$ \\
\hline & 13 & CSE: Brain damage $(\mathrm{F})$ & $38(71)$ & $52(98)$ & $14.43 * * *$ \\
\hline & 10 & CSE: Permanent memory impairment $(\mathrm{F})$ & $25(47)$ & $46(87)$ & $3.51 * * *$ \\
\hline & 12 & CSE: Broken bones $(\mathrm{F})$ & $48(90)$ & $52(98)$ & $2.83 * * *$ \\
\hline & 14 & CI: Dementia (F) & $23(43)$ & $37(70)$ & $8.91 * *$ \\
\hline & 4 & Conclusive evidence exists for the efficacy of ECT in the treatment of depression (T) & $40(75)$ & $52(98)$ & $3.48 * *$ \\
\hline & 15 & CI: A known pregnancy $(\mathrm{F})$ & $31(59)$ & $46(87)$ & $2.97 * *$ \\
\hline & 11 & CSE: Headache (T) & $4(8)$ & $14(26)$ & $1.24 *$ \\
\hline & 8 & IND: Depression (T) & $43(81)$ & $52(98)$ & $0.24 *$ \\
\hline & 2 & Patients must stop all medication before they can be given ECT $(\mathrm{F})$ & $37(70)$ & $47(89)$ & $1.26^{*}$ \\
\hline & 7 & Voltage used is in the order of 500 volts $(F)$ & $18(34)$ & $29(55)$ & $0.45^{*}$ \\
\hline & 3 & Relatives need to give consent before ECT can be given $(\mathrm{F})$ & $12(23)$ & $10(19)$ & 9.82 \\
\hline & 16 & CI: Brain tumours $(\mathrm{F})$ & $25(47)$ & $22(42)$ & 6.66 \\
\hline & 5 & Patients cannot be given ECT against their will $(\mathrm{F})$ & $48(91)$ & $50(94)$ & 2.13 \\
\hline & 9 & IND: Anorexia nervosa $(\mathrm{F})$ & $12(23)$ & $18(34)$ & 1.78 \\
\hline & 6 & Patients need to have nil by mouth from the night before ECT $(\mathrm{T})$ & $8(15)$ & $5(10)$ & 0.14 \\
\hline & 1 & ECT should only be given to a patient who can eat and drink adequately $(\mathrm{F})$ & $31(59)$ & $27(51)$ & 0.13 \\
\hline
\end{tabular}

QuAKE, Questionnaire on Attitudes and Knowledge of ECT; F, false; $T$, true; CSE, common side effect; IND, indication; $C I$, contraindication

${ }^{\mathrm{a}} \mathrm{df}=52 ; p$ values adjusted for multiple comparisons (Bonferroni significance threshold $=0.003$ )

${ }^{\mathrm{b}}$ Cells represent items answered correctly; $\mathrm{df}=1 ; p$ values calculated using the McNemar test

${ }^{\dagger}$ Indicates reverse-coded

${ }^{*} p<0.05 ; * * p<0.01 ; * * * p<0.001$

end, we used about one-third of the overall video content available. Depending on the specific needs and training level of target learners, the available content could be adjusted differently.

The materials were well received by learners, who saw ECT "come alive" in a way that a traditional lecture could not, and with logistic and practical ease that is not often possible for in-person visits to an ECT suite. Moreover, even if having such access, learners are unlikely to follow a patient from time of consent, through treatment, and into a follow-up phase of treatment. Apart from permitting such compression of an elusive longitudinal experience, the video-enhanced 
educational approach has the advantage of offering all learners in a class a standardized educational experience. Finally, training programs without local ECT expertise can provide the same level of training by taking advantage of these resources.

Following the video-enriched training intervention, student surveys demonstrated clear improvements in attitudes and knowledge related to ECT, with attitudinal change being the stronger of the two. Pre-intervention knowledge of ECT was greater than expected, limiting the potential effect size of the intervention on the knowledge outcome, and yielding effects that are statistically significant but not clinically meaningful.

Given that these portrayals are true to best practices and were developed following explicit educational objectives, they offer advantages over many of the materials available online. Online resources can be either inaccurate and contribute to entrenching stigmatized views of mental illnesses and ECT, or accurate and even "patient forward" but lacking the depth and context appropriate for most healthcare trainees.

\section{Means of Delivery: Synchronous Videoconferencing}

Prior to the pandemic, there had certainly been many efforts to enhance online-delivered education. Asynchronous platforms such as Coursera are by now well established and reach a large student viewership around the world; universities and professional organizations have rich asynchronous offerings. Synchronous education has been less broadly implemented, especially in medical education or across large time zone differences. There are promising early examples of effective synchronous approaches within healthcare, including in medical education [22] and in qualitative research [23].

The 2020 pandemic accelerated the adoption of synchronous videoconferencing for a wide range of educational activities, including classroom teaching. We implemented our study using the Zoom platform, which was nimble and easy to implement, with specific guidelines relevant to its application during the pandemic [24]. Specifically, the quality of video and audio delivery was seamless, as was the ability to interact with a group of sixty students in real time and across 11 time zones.

The COVID-19 pandemic forced us to embrace a means of content delivery we had not anticipated. To our pleasant surprise, we found synchronous videoconferencing to be a feasible interface. Beyond the sharing of curricular initiatives and the dissemination of enduring didactic material such as our videos, we anticipate that synchronous videoconferencing may have a role to last well beyond the current crisis. Specifically, we consider that this approach could have great potential in building and strengthening educational networks: by reaching out to smaller programs and those in remote and isolated areas, as well as by helping to better connect large urban programs stretched across multiple sites that make for challenging in-person meetings at dedicated locations. In short, we are hopeful that a situation borne out of social isolation may provide enduring lessons to better interconnect us and our educational efforts in the years to come.

We recognize several limitations to our study. First, regarding generalizability, our study included only medical students in one class at one school learning from one instructor; implementation at other institutions or for other levels of learners (e.g., psychiatry residents or other health professionals) may require adjustments, and findings could be different. Second, we recognize the possibility of a social desirability bias: students may have answered the surveys more favorably at the second time point. Third, we selected the QuAKE as our outcome measure, but recognize that there are other instruments $[25,26]$ that may have been differentially sensitive to change or led to different findings. Fourth, we did not have a control group, such as of students exposed to the lecture content but not to the videos, which could have helped identify the active components of the intervention. Finally, we recognize that even as the effect of our intervention led to changes in attitudes and knowledge, we do not know whether these effects will prove to be durable. A single exposure may not be sufficient to have an enduring effect; a spiral curriculum could be beneficial, wherein repeat encounters with ECT as a treatment modality would reinforce previous learning.

In conclusion, the video-based educational materials that we used proved easy to implement in a virtual classroom, were amenable to adaptation by end-use instructors, were well received by learners, and led to measurable changes in their knowledge of and attitudes toward ECT.

Acknowledgments We appreciate the contributions of the professional actors, the logistic support of Barbara Hildebrand at the Teaching and Learning Center, and the students' engaged participation. The authors have informed the journal that they agree that both Brandon Kitay and Andrés Martin completed the intellectual and other work typical of the first author.

Funding Information Supported by a grant from the Association of Directors of Medical Student Education in Psychiatry awarded to Brandon Kitay, by the Riva Ariella Ritvo Endowment at the Yale School of Medicine, and by NIMH R25 MH077823, Research Education for Future Physician-Scientists in Child Psychiatry.

\section{Compliance with Ethical Standards}

The Yale Human Investigations Committee (Protocol no. 2000026843) and the Tel-Aviv University Institutional Review Board (Protocol no. 0000946-1) approved the study and deemed it exempt, with survey completion standing for tacit consent.

Disclosures On behalf of all authors, the corresponding author states that there is no conflict of interest. 


\section{References}

1. Wilkinson ST, Rosenheck RA. Electroconvulsive therapy at a veterans health administration medical center. J ECT. 2017;33:24952 .

2. Wilkinson ST, Agbese E, Leslie DL, Rosenheck RA. Identifying recipients of electroconvulsive therapy: data from privately insured Americans. Psychiatr Serv. 2018;69:542-8.

3. Jaffe R. The practice of electroconvulsive therapy: recommendations for treatment, training, and privileging: a task force report of the American Psychiatric Association. 2nd ed. Philadelphia: American Psychiatric Press; 2002.

4. ADMSEP (Association of Directors of Medical Student Education in Psychiatry). Clinical learning objectives guide for psychiatry education of medical students [Internet]. 2007. Available from: https://www.admsep.org/pdf/templates/Clinical_LO_Guide_ Outline_Format_Nov_07.pdf. Accessed 29 Jul 2020

5. Dolenc TJ, Philbrick KL. Achieving competency in electroconvulsive therapy: a model curriculum. Acad Psychiatry. 2007;31:65-7.

6. Dinwiddie SH, Spitz D. Resident education in electroconvulsive therapy. J ECT. 2010;26:310-6.

7. Williams NR, Taylor JJ, Snipes JM, Short EB, Kantor EM, George MS. Interventional psychiatry: how should psychiatric educators incorporate neuromodulation into training? Acad Psychiatry. 2014;38:168-76.

8. Clothier JL, Freeman T, Snow L. Medical student attitudes and knowledge about ECT. J ECT. 2001;17:99-101.

9. Walter G, McDonald A, Rey JM, Rosen A. Medical student knowledge and attitudes regarding ECT prior to and after viewing ECT scenes from movies. J ECT. 2002;18:43-6.

10. Sienaert P. Based on a true story? The portrayal of ECT in international movies and television programs. Brain Stimul. Elsevier Inc. 2016;9:882-91.

11. Aoki Y, Yamaguchi S, Ando S, Sasaki N, Bernick PJ, Akiyama T. The experience of electroconvulsive therapy and its impact on associated stigma: a meta-analysis. Int J Soc Psychiatry. 2016;62: 708-18.

12. Andrews M, Hasking P. Effect of two educational interventions on knowledge and attitudes towards electroconvulsive therapy. J ECT. 2004;20:230-6.

13. Trenton A, Pelchat R. Medical students' perceptions of electroconvulsive therapy: the impact of direct exposure. J ECT. 2016;32:20 2.

14. Battersby M, Ben-tovim D, Eden J. Electroconvulsive therapy: a study of attitudes and attitude change after seeing an educational video. Aust N Z J Psychiatry. 1993;27:613-9.
15. Shah N, Averill PM. Third-year medical students' understanding, knowledge, and attitudes toward the use of electroconvulsive therapy: a pre-exposure and postexposure survey. J ECT. 2009;25:2614.

16. Warnell RL, Duk AD, Christison GW, Haviland MG. Teaching electroconvulsive therapy to medical students: effects of instructional method on knowledge and attitudes. Acad Psychiatry. 2005;29:433-6.

17. Tsai J, Huang M, Wilkinson ST, Edelen C. Effects of video psychoeducation on perceptions and knowledge about electroconvulsive therapy. Psychiatry Res. Elsevier Ireland Ltd. 2020;286: 112844.

18. Genc ES, Wu HE, Pinjari OF, Fernandez LA, Henchcliffe BE, Husain M, et al. Image of electroconvulsive therapy in YouTube videos. J ECT. 2020;00:1.

19. Lewis KL, Bohnert CA, Gammon WL, Hölzer H, Lyman L, Smith $\mathrm{C}$, et al. The Association of Standardized Patient Educators (ASPE) Standards of Best Practice (SOBP). Adv Simul Advances in Simulation. 2017;2:1-8.

20. Lutchman RD, Stevens T, Bashir A, Orrell M. Mental health professionals' attitudes towards and knowledge of electroconvulsive therapy. J Ment Health. 2001;10:141-50.

21. Adedokun O, Burgess W. Analysis of paired dichotomous data: a gentle introduction to the McNEmar test in SPSS. J Multidiscip Educ. 2012;8:125-31.

22. Hortos K, Sefcik D, Wilson SG, McDaniel JT, Zemper E. Synchronous videoconferencing: impact on achievement of medical students. Teach Learn Med. 2013;25:211-5.

23. Archibald MM, Ambagtsheer RC, Casey MG, Lawless M. Using zoom videoconferencing for qualitative data collection: perceptions and experiences of researchers and participants. Int J Qual Methods. 2019;18:1-8.

24. Zoom. Support during the COVID-19 pandemic [Internet]. Available from: https://zoom.us/docs/en-us/covid19.html. Accessed 29 Jul 2020

25. Alexander L, Malone K, Counihan E, Kennedy J, Roddy D, Delaney L. Assessing public attitudes to electroconvulsive therapy: validation of the Modified ECT Attitudes Questionnaire using a systematic analysis. J ECT. 2020;36:47-53.

26. Tsai J, Huang M, Wilkinson ST, Edelen C, Rosenheck RA, Holtzheimer PE. A measure to assess perceptions and knowledge about electroconvulsive therapy: development and psychometric properties. J ECT. 2020;36:E1-6.

Publisher's Note Springer Nature remains neutral with regard to jurisdictional claims in published maps and institutional affiliations. 